\title{
Anomalous couplings in associated $V H$ production with Higgs boson decay to massive $b$ quarks at NNLO in QCD
}

\author{
Wojciech Bizoń, ${ }^{1,2, *}$ Fabrizio Caola, ${ }^{3, \dagger}$ Kirill Melnikov, ${ }^{1, \ddagger}$ and Raoul Röntsch $\oplus^{4, \S}$ \\ ${ }^{1}$ Institute for Theoretical Particle Physics, KIT, Karlsruhe 76128, Germany \\ ${ }^{2}$ Institute for Astroparticle Physics, KIT, Karlsruhe 76021, Germany \\ ${ }^{3}$ Rudolf Peierls Centre for Theoretical Physics, Clarendon Laboratory, Parks Road, Oxford OX1 3PU, UK \\ and Wadham College, Oxford OX1 3PN, United Kingdom \\ ${ }^{4}$ Theoretical Physics Department, CERN, 1211 Geneva 23, Switzerland
}

(Received 19 August 2021; accepted 10 January 2022; published 26 January 2022)

\begin{abstract}
We combine the NNLO QCD description of Higgs boson production in association with an electroweak vector boson $V=W$ or $Z$ with a similarly precise description of Higgs boson decays into a pair of massive $b$ quarks and with the anomalous couplings that modify interactions of the Higgs and electroweak vector bosons. The resulting numerical code provides the most advanced theoretical tool to investigate such anomalous couplings in the associated Higgs boson production process. We study the impact of anomalous couplings on fiducial cross sections and differential distributions and argue that, with increased QCD precision, smaller anomalous couplings become accessible in kinematic regions where the effects of higher-dimensional operators in the Standard Model effective field theory remain small and the effective field theory expansion is under control.
\end{abstract}

DOI: $10.1103 /$ PhysRevD.105.014023

\section{INTRODUCTION}

Studies of Higgs boson properties in experiments at the Large Hadron Collider (LHC) have converged to the conclusion [1] that the Standard Model of particle physics describes Higgs couplings to gauge bosons and to (some) matter fields with a precision between $10 \%$ and $30 \%$. To reach a higher precision, new experimental measurements as well as refined theoretical descriptions of major Higgs production processes are needed. The forthcoming Run III of the LHC, as well as its high-luminosity phase, will play an important role in achieving these goals.

From a theoretical perspective, some of the simplest but also most interesting Higgs boson production processes are those where Higgs bosons are produced in association with vector bosons, i.e., $p p \rightarrow W H$ and $p p \rightarrow Z H$. Indeed, at lowest order in perturbative QCD, both of these processes are of the Drell-Yan type $p p \rightarrow V^{*} \rightarrow V H$, so that their description through next-to-next-to-leading order (NNLO) in perturbative $\mathrm{QCD}$ is quite straightforward. These

\footnotetext{
*wojciech.bizon@kit.edu

fabrizio.caola@physics.ox.ac.uk

kirill.melnikov@kit.edu

§raoul.rontsch@cern.ch
}

Published by the American Physical Society under the terms of the Creative Commons Attribution 4.0 International license. Further distribution of this work must maintain attribution to the author(s) and the published article's title, journal citation, and DOI. Funded by SCOAP ${ }^{3}$. production processes allow for a study of Higgs-gauge interactions.

Associated production processes also provide an environment in which the decay of the Higgs to a $b \bar{b}$ pair can be observed [2-6], allowing the study of the Higgs coupling to $b$ quarks. Therefore, describing both the production $p p \rightarrow$ $V H$ and the decay $H \rightarrow b \bar{b}$ processes with the same precision is important. Moreover, it is important to consider $b$ quarks as massive since in this case one can apply conventional jet algorithms to identify $b$ jets and reconstruct Higgs boson kinematics. Indeed, it was shown recently in Ref. [7] that working with massless $b$ quarks may lead to sizeable differences in theoretical predictions for the associated production process $p p \rightarrow W H$.

The status of theoretical predictions for $\mathrm{VH}$ processes in the Standard Model (SM) is quite advanced. Refined predictions that include both QCD [8-23] and electroweak [24-26] radiative corrections are available. Recently, NNLO QCD corrections for $W H$ production in association with a hard jet were computed [27].

A major difference between the $W H$ and $Z H$ final states is that, starting from NNLO QCD, the latter receives large contributions from the $g g \rightarrow Z H$ process. ${ }^{1}$ The $\mathcal{O}\left(\alpha_{s}^{2}\right)$ contribution of this process has been known for a long time $[29,30]$. Approximate results for the $\mathcal{O}\left(\alpha_{s}^{3}\right)$ contributions due to $g g \rightarrow \mathrm{ZH}$ suggest that these can be quite

\footnotetext{
${ }^{1}$ Apart from its relevance in exploring the Higgs sector, this process can also provide insight into the $Z b \bar{b}$ interactions [28].
} 
large [31,32] and should be included for reliable predictions despite being formally subleading. In the recent past, significant effort went into their computation [33-36], using either numerical methods or phenomenologically motivated analytic approximations. Strategies to extract the $g g \rightarrow Z H$ contribution from experimental data have been investigated in Ref. [37]. Both the $W H$ [38,39] and $Z H$ [39-41] processes have been matched to parton shower Monte Carlo tools retaining NNLO QCD accuracy. Reference [41] also includes NNLO QCD corrections to the $H \rightarrow b \bar{b}$ decay. Dedicated parton shower Monte Carlo tools including both next-to-leading order (NLO) QCD and electroweak corrections [42] and a refined treatment of the $g g \rightarrow Z H$ contribution $[43,44]$ also exist.

Similarly, advanced theoretical predictions for the $H \rightarrow$ $b \bar{b}$ decay are available. The total rate is known up to $\mathrm{N}^{4} \mathrm{LO}$ QCD in the limit of massless bottom quarks [45-53]. Electroweak corrections are also known [54-57] as are the mixed QCD-electroweak corrections [58]. A comprehensive review of computations of the $H \rightarrow b \bar{b}$ inclusive branching ratio and its uncertainties can be found in Refs. [59,60]. At the differential level, QCD corrections for massless $b$ quarks are known at NNLO [22,61-63] and $\mathrm{N}^{3} \mathrm{LO}[64,65]$. NNLO QCD results retaining the full $b$-quark mass dependence have been presented in Refs. [7,66-68]. Top-quark effects have been studied in Refs. [69,70].

Given this level of sophistication, it is interesting to extend the precise modeling of associated Higgs boson production and $H \rightarrow b \bar{b}$ decay to cases where Higgs couplings to gauge and matter fields differ from the ones in the Standard Model Lagrangian. A convenient way to do this is provided by the Standard Model effective field theory (SMEFT), see e.g., Ref. [71] for a review. In principle, one may aim at the complete description of the processes $p p \rightarrow V H(b \bar{b})$ in the SMEFT, taking into account contributions of all dimension-six operators present in the SMEFT Lagrangian. However, since in this case the number of operators that one has to consider becomes quite large, it makes sense to first restrict oneself to a subset of operators. A particularly interesting choice is those that modify the couplings of the Higgs boson to electroweak gauge bosons (for a recent study, see e.g., Ref. [72]). Indeed, this approach has been adopted in Ref. [73] where this process was studied with NLO QCD accuracy. The main goal of this work is to promote this analysis to full NNLO QCD.

The paper is organized as follows. In Sec. II we briefly describe the computation of NNLO QCD corrections to the processes $p p \rightarrow V H(b \bar{b})$, with $V=W, Z$, in the Standard Model. Such a computation for the $W H$ final state was discussed earlier in Ref. [7]; the results for $Z H(b \bar{b})$ with massive $b$ quarks are new. In Sec. III we describe calculations that include both anomalous couplings and NNLO QCD corrections to Higgs boson associated production and its decay into a $b \bar{b}$ pair. We consider scenarios where anomalous couplings modify fiducial cross sections only slightly so that the availability of highly precise theoretical predictions for fiducial cross sections and kinematic distributions becomes important. We conclude in Sec. IV.

\section{ASSOCIATED PRODUCTION $p p \rightarrow V H(b \bar{b})$ IN THE STANDARD MODEL}

In this section, we briefly describe the computation of NNLO QCD radiative corrections to the associated production process $p p \rightarrow V H(b \bar{b})$ in the Standard Model, keeping the $b$-quark masses nonzero. We note that the $W H(b \bar{b})$ final state was discussed recently in Ref. [7]. The results for the $Z H(b \bar{b})$ final state, which we mostly focus on in this section, are new.

As we already mentioned in Sec. I, the computation of NNLO QCD radiative corrections to $p p \rightarrow V H(b \bar{b})$ involves two major ingredients: QCD corrections to the production process $p p \rightarrow V H$ and QCD corrections to the decay process $H \rightarrow b \bar{b}$. An earlier computation of NNLO QCD corrections to $p p \rightarrow W H$ with the decay $H \rightarrow b \bar{b}$ for massless $b$ quarks was described in Ref. [22]. This computation was based on the nested soft-collinear subtraction scheme introduced in Ref. [74], and employed simple analytic formulas derived for the production and decay processes of color-singlet states in Refs. [63,75].

This earlier computation was recently extended by including Higgs boson decays to massive $b$ quarks [7], using predictions for $H \rightarrow b \bar{b}$ decay from Ref. [67] and modifying the calculation of NNLO QCD corrections to the production process in Ref. [22] to exclude $b$ quarks from being active partons in a proton. As we already mentioned in Sec. I, working with massive quarks allows us to employ conventional jet algorithms to describe $b$-flavored jets.

In this paper, we have extended the above computations to the $\mathrm{ZH}$ final state. From the point of view of soft and collinear subtractions, such an extension is straightforward since the analytic formulas derived in Ref. [75] are applicable to all color-singlet final states. For this reason, a transition from the $W H$ final state to the $Z H$ final state only requires us to change the relevant matrix elements and adjust flavors of colliding partons. However, an important difference between $\mathrm{ZH}$ and $\mathrm{WH}$ final states is the contribution of the $g g \rightarrow \mathrm{ZH}$ partonic process which only exists in the former case. Thanks to a large gluon flux, this contribution is significant; accounting for it in the theoretical prediction for the $Z H$ final state is important, especially for large values of $Z H$ invariant masses. In our calculation, we have included the exact $\mathcal{O}\left(\alpha_{s}^{2}\right)$ contributions to the $g g \rightarrow Z H$ channel but we have neglected higher-order terms which so far are not available. Apart from corrections to the $g g$ channel, there are other classes of contributions proportional to the top Yukawa coupling for 
which the exact two-loop amplitudes are unknown. Here we followed the approach of Ref. [20], which is based on the analysis of Ref. [15]. In the notation of Ref. [15], we have included the so-called $V_{\mathrm{I}, \mathrm{II}}$ contributions keeping only the leading term in the $m_{\text {top }} \rightarrow \infty$ expansion. We have also included exact $R_{\mathrm{I}}$ contributions, but discarded $R_{\mathrm{II}}$ terms since they have been shown to be very small for phenomenologically relevant setups [15]. Similarly, we have neglected effects of top quark loops in Drell-Yan type diagrams $p p \rightarrow V^{*}, V^{*} \rightarrow V H$ as they too have negligible phenomenological impact [15].

As the first step in our discussion, we present cross sections and differential distributions for the two associated production processes

$$
\begin{gathered}
p p \rightarrow W^{+} H \rightarrow\left(\nu_{e} e^{+}\right)(b \bar{b}), \\
p p \rightarrow Z H \rightarrow\left(e^{-} e^{+}\right)(b \bar{b}),
\end{gathered}
$$

at the $13 \mathrm{TeV}$ LHC. We treat both decay processes $V \rightarrow \ell \bar{\ell}$ and $H \rightarrow b \bar{b}$ in the narrow-width approximation. Following Ref. [7], we write differential cross sections as

$\mathrm{d} \sigma_{p p \rightarrow V H(b \bar{b})} \propto \operatorname{Br}(H \rightarrow b \bar{b}) \times \mathrm{d} \sigma_{p p \rightarrow V H} \times \frac{\mathrm{d} \Gamma_{H \rightarrow b \bar{b}}}{\Gamma_{H \rightarrow b \bar{b}}}$,

and we do not perform an expansion of $\operatorname{Br}(H \rightarrow b \bar{b})$ in a series of $\alpha_{s}$, treating it as an input parameter.

For numerical computations we take $\operatorname{Br}(H \rightarrow b \bar{b})=$ 0.5824 as recommended by the Higgs Cross Section Working Group [76]. We set the Higgs boson mass to $M_{H}=125 \mathrm{GeV}$, the vector boson masses to $M_{W}=$ $80.399 \mathrm{GeV}$ and $M_{Z}=91.1876 \mathrm{GeV}$, respectively, the on-shell $b$-quark mass to $m_{b}=4.78 \mathrm{GeV}$, and the top quark mass to $m_{t}=173.2 \mathrm{GeV}$. We use the Fermi constant $G_{F}=1.16639 \times 10^{-5} \mathrm{GeV}^{-2}$ and the weak mixing angle $\sin ^{2} \theta_{W}=0.2226459$. The widths of vector bosons are taken to be $\Gamma_{W}=2.1054 \mathrm{GeV}$ and $\Gamma_{Z}=2.4952 \mathrm{GeV}$, while the Higgs width is taken to be $\Gamma_{H}=4.165 \mathrm{MeV}$. Finally, we take the CKM matrix to be diagonal.

We note that the $b$-quark Yukawa coupling that enters the $H \rightarrow b \bar{b}$ decay rate is computed using the $\overline{\mathrm{MS}}$ $b$-quark mass calculated at $\mu=M_{H}, \quad \bar{m}_{b}\left(\mu=M_{H}\right)=$ $2.81 \mathrm{GeV}[77,78]$. However, since the physical cross sections in Eq. (3) are proportional to the ratio $\mathrm{d} \Gamma_{H \rightarrow b \bar{b}} / \Gamma_{H \rightarrow b \bar{b}}$, the dependence on the Yukawa coupling to a large extent cancels out in the results that are presented below.

We define $W^{+} H$ and $Z H$ final states using kinematic selection criteria for charged leptons and $b$-flavored jets. To this end, we require that an event contains at least two $b$ jets that are defined with the anti- $k_{t}$ jet algorithm $[79,80]$ and we choose the jet radius $R=0.4$. Pseudorapidities and transverse momenta of charged leptons and $b$ jets should satisfy the following constraints

$$
\begin{aligned}
\left|\eta_{l}\right|<2.5, & p_{t, l}>25 \mathrm{GeV}, \\
\left|\eta_{j_{b}}\right|<2.5, & p_{t, j_{b}}>20 \mathrm{GeV} .
\end{aligned}
$$

Finally, following experimental analyses, we may additionally require that the vector boson has large transverse momentum, $p_{t, V}>150 \mathrm{GeV}$. We always state explicitly when this cut is applied.

The fiducial cross sections are calculated with the four-flavor parton distribution function (PDF) set NNPDF31_nnlo_as_0118_nf_4. We emphasize that we employ NNLO PDFs to compute LO, NLO and NNLO cross sections in what follows. Moreover, we use $\alpha_{s}\left(M_{Z}\right)=0.118$ and perform the running of the strong coupling at three loops with five active flavors.

For all numerical results presented in this paper, the central value of the renormalization and factorization scales in the production process is set to one half of the invariant mass of the $V H$ system, i.e., $\mu_{r}=\mu_{f}=\mu=\frac{1}{2} \sqrt{\left(p_{V}+p_{H}\right)^{2}}$. The renormalization scale for the decay process is set to the Higgs boson mass, $\mu_{r \text {,dec }}=M_{H}$; it is kept fixed for all results reported in this paper. The uncertainty of the cross sections is obtained by varying the scale in the production process by a factor of two around the central value.

We present fiducial cross sections for the above set of cuts at leading order (LO), next-to-leading order (NLO) and NNLO in QCD for the $W^{+} H$ and $Z H$ production processes in Tables I and II. The contribution of the loop-induced gluon-initiated process $g g \rightarrow Z H$ is reported separately (this contribution does not include the Drell Yan-like $g g \rightarrow$ $\mathrm{ZH}+\mathrm{X}$ processes, which are included in the NNLO results). As we already mentioned, this contribution is rather large. Indeed, it follows from Tables I and II that it increases the fiducial cross section by $15 \%$ if no cut on the $Z$ transverse momentum is applied, and by about $25 \%$ if the

TABLE I. Fiducial cross sections for $p p \rightarrow W^{+} H \rightarrow$ $\left(\nu_{e} e^{+}\right)(b \bar{b})$ and $p p \rightarrow Z H \rightarrow\left(e^{-} e^{+}\right)(b \bar{b})$ at the $13 \mathrm{TeV}$ LHC at various orders of QCD perturbation theory calculated with massive $b$ quarks. We set the factorization and renormalization scales equal to each other, $\mu_{r}=\mu_{f}=\mu$. We use $\mu=$ $\frac{1}{2} \sqrt{\left(p_{V}+p_{H}\right)^{2}}$ for the central value and the uncertainties are calculated by varying the scale $\mu$ by a factor of two in both directions. See text for details.

\begin{tabular}{lllc}
\hline \hline Order & $\sigma_{\text {fid }}^{W^{+} H}(\mathrm{fb})$ & $\sigma_{\text {fid }}^{Z H}(\mathrm{fb})$ & $\sigma_{\text {fid }}^{Z H+g g Z H}(\mathrm{fb})$ \\
\hline LO & $21.22_{-0.95}^{+0.76}$ & $5.13_{-0.21}^{+0.17}$ & \\
NLO & $24.48_{-0.25}^{+0.22}$ & $5.87_{-0.06}^{+0.10}$ & \\
NNLO & $23.86_{-0.17}^{+0.16}$ & $5.69_{-0.03}^{+0.03}$ & $6.52_{-0.19}^{+0.25}$ \\
\hline \hline
\end{tabular}


TABLE II. Fiducial cross sections in the boosted region $\left(p_{t, V}>150 \mathrm{GeV}\right)$ for $p p \rightarrow W^{+} H \rightarrow\left(\nu_{e} e^{+}\right)(b \bar{b})$ and $p p \rightarrow$ $Z H \rightarrow\left(e^{-} e^{+}\right)(b \bar{b})$ at the $13 \mathrm{TeV}$ LHC at various orders of QCD perturbation theory calculated with massive $b$ quarks. We set the factorization and renormalization scales equal to each other, $\mu_{r}=\mu_{f}=\mu$. We use $\mu=\frac{1}{2} \sqrt{\left(p_{V}+p_{H}\right)^{2}}$ for the central value and the uncertainties are calculated by varying the scale $\mu$ by a factor of two in both directions. See text for details.

\begin{tabular}{lccc}
\hline \hline Order & $\sigma_{\text {fid }}^{W^{+} H}(\mathrm{fb})$ & $\sigma_{\text {fid }}^{Z H}(\mathrm{fb})$ & $\sigma_{\text {fid }}^{Z H+g g Z H}(\mathrm{fb})$ \\
\hline LO & 3.89 & 0.97 & \\
NLO & $4.79_{-0.10}^{+0.13}$ & $1.20_{-0.03}^{+0.03}$ & \\
NNLO & $4.79_{-0.06}^{+0.02}$ & $1.22_{-0.03}^{+0.03}$ & $1.52_{-0.09}^{+0.11}$ \\
\hline \hline
\end{tabular}

$Z$ boson is required to have a transverse momentum in excess of $150 \mathrm{GeV}^{2}$

We also see from Tables I and II that uncertainties of quark-initiated contributions at NNLO are less than a percent if no $p_{t, V}$ cut is applied, and around $2 \%$ in the presence of this cut. The inclusion of the $g g \rightarrow \mathrm{ZH}$ contribution increases the uncertainty significantly, to about $4 \%$ without the additional $p_{t, V}$ cut and to about $7 \%$ if we require $p_{t, V}>150 \mathrm{GeV}$. To reduce this uncertainty, the $g g \rightarrow \mathrm{ZH}$ contribution has to be computed at NLO in perturbative QCD and, as we already mentioned in Sec. I, a significant effort in this direction is currently underway [33-36].

Before we turn to the discussion of anomalous couplings, we show a few kinematic distributions in the Standard Model. Since we have discussed the $W^{+} H$ process in some detail earlier [7], we focus exclusively on the distributions for the $p p \rightarrow Z H$ process.

In Fig. 1 we display the invariant mass of the Higgs boson and $Z$ boson system. We note that the invariant mass is reconstructed from the "true" Higgs boson momentum $p_{H}$ and the $Z$-boson momentum $p_{Z}$, i.e., $M_{Z H}^{2}=\left(p_{H}+p_{Z}\right)^{2}$; however, to be included in the plot, an event is required to pass the kinematic cuts described above. We observe large changes in this distribution starting at $M_{Z H} \sim 350 \mathrm{GeV}$ where the $g g \rightarrow Z H$ contribution becomes significant. However, the NNLO QCD corrections to the quark-initiated processes change the NLO QCD distribution only slightly; they are about $-5 \%$ at low invariant masses and become slightly positive at larger $M_{Z H}$ values.

The transverse momentum and rapidity distributions of the Higgs boson are shown in Figs. 2 and 3, respectively.

\footnotetext{
${ }^{2}$ We note that in the $g g \rightarrow Z H$ channel there is a strong cancellation between the box and triangle diagrams, which however is only active for SM couplings. If e.g., the top Yukawa coupling were to be different, a very strong enhancement of this contribution could be expected [81]. For example, if the top Yukawa coupling had the opposite sign [44,82-84], the NNLO cross section in Table I would increase to about $10 \mathrm{fb}$.
}

In these plots, the Higgs momentum is reconstructed from two $b$ jets as described earlier. We note that if more than two $b$ jets appear in the final state, we choose the pair whose invariant mass is closest to the Higgs boson mass $M_{H}=125 \mathrm{GeV}$. The corrections to the Higgs transverse momentum distribution show a pattern that is similar to what is seen in the $\mathrm{ZH}$ invariant mass distribution. Indeed, in the case of quark-initiated $\mathrm{ZH}$ production, the NNLO QCD corrections are negative and decrease NLO distributions by no more than $5 \%$, whereas if $g g \rightarrow \mathrm{ZH}$ is included in the theoretical prediction, there are large modifications of the $p_{t, H}$ distribution starting at $p_{t, H} \sim 150 \mathrm{GeV}$.

Kinematic distributions that are integrated over the Higgs boson transverse momentum and $\mathrm{ZH}$ invariant masses do not exhibit local enhancements due to the $g g \rightarrow$ $\mathrm{ZH}$ process but, rather, show an overall increase similar to fiducial cross sections. This is the case for e.g., the (reconstructed) Higgs rapidity distribution shown in Fig. 3; we observe there that with or without $g g \rightarrow \mathrm{ZH}$ contributions, the rapidity distribution is modified by an almost constant $K$-factor.

Finally, we give an example of a kinematic distribution that exhibits large NNLO QCD corrections that are not related to the $g g \rightarrow Z H$ process. In Fig. 4 we display the Higgs boson invariant mass distribution where the Higgs boson is reconstructed from two $b$ jets whose invariant mass is the closest to Higgs boson mass. The $g g \rightarrow \mathrm{ZH}$ process contributes only to the $M_{H(b \bar{b})}=M_{H}$ bin since it has at most two $b$ jets in the final state and the invariant mass of these $b$ jets is equal to $M_{H}$. However, similar to the case of the $W^{+} H$ final state discussed in Ref. [7], we observe very large NNLO QCD effects in the $M_{H(b \bar{b})}$ distributions away from the peak at the true mass of the Higgs boson due to initial- and final-state radiation.

\section{ASSOCIATED VH PRODUCTION WITH ANOMALOUS COUPLINGS}

Theoretical predictions for the associated production processes can be modified by both higher-order QCD effects and by contributions of physics beyond the Standard Model (BSM). Under certain circumstances, the latter can be described by an effective Lagrangian that parametrizes possible deviations from the Standard Model in terms of operators with increasing mass dimensions. A convenient description is provided by the so-called Standard Model effective field theory (SMEFT), see Ref. [71] for a review.

The main aim of this paper is to study the interplay between higher order QCD corrections and anomalous Higgs interactions. For this reason, and with an eye on comparing with and extending previous results in the literature, we follow Ref. [73] which performs this analysis at NLO in QCD. We therefore adopt the same formalism as this reference by only considering operators that modify 

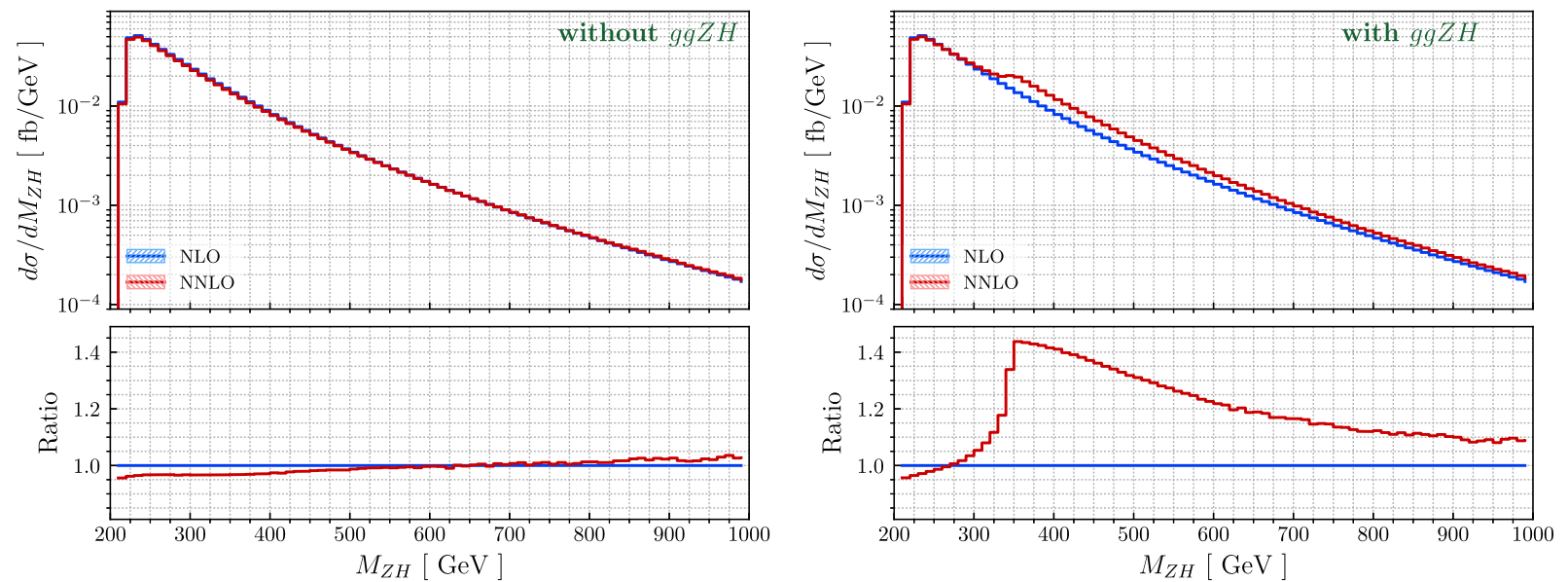

FIG. 1. The invariant mass $M_{Z H}$ distribution at NLO (blue) and NNLO (red) at the $13 \mathrm{TeV}$ LHC with the fiducial cuts discussed in the text. We present the NNLO results without (left) and with (right) the $g g \rightarrow Z H$ contribution. We display results for the central scale $\mu=\frac{1}{2} \sqrt{\left(p_{V}+p_{H}\right)^{2}}$. The lower panes show the ratio of the NNLO results to the NLO ones. See text for details.
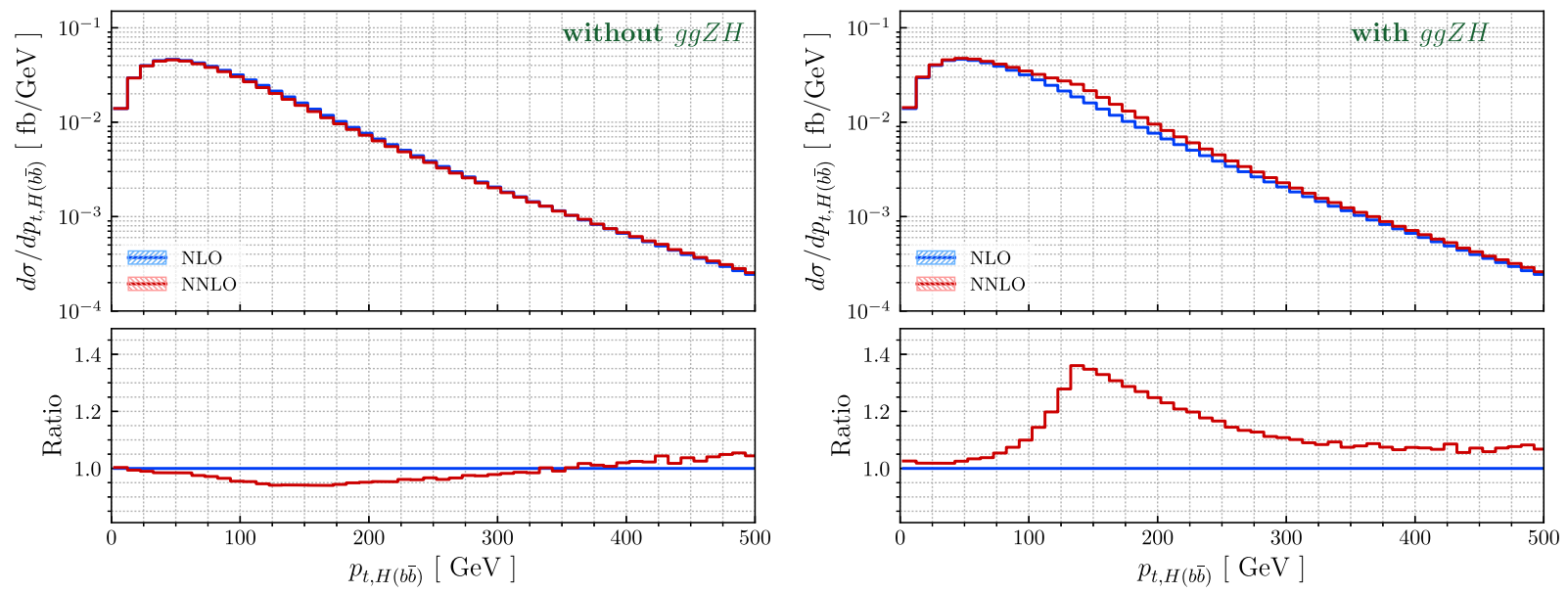

FIG. 2. The transverse momentum distribution of the reconstructed Higgs boson at NLO (blue) and NNLO (red) at the 13 TeV LHC with the fiducial cuts discussed in the text. We present the NNLO results without (left) and with (right) the $g g \rightarrow Z H$ contribution. We display results for the central scale $\mu=\frac{1}{2} \sqrt{\left(p_{V}+p_{H}\right)^{2}}$. The lower panes show the ratio of the NNLO results to the NLO ones. See text for details.

interactions of the Higgs boson to electroweak gauge bosons. The part of the SMEFT Lagrangian that is relevant to us

$$
\begin{aligned}
\mathcal{L}_{\text {anom }}= & -\frac{1}{4 \Lambda} g_{h z z}^{(1)} Z_{\mu \nu} Z^{\mu \nu} h-\frac{1}{2 \Lambda} g_{h w w}^{(1)} W^{\mu \nu} W_{\mu \nu}^{\dagger} h \\
& -\frac{1}{\Lambda} g_{h z z}^{(2)} Z_{\nu} \partial_{\mu} Z^{\mu \nu} h-\frac{1}{\Lambda}\left[g_{h w w}^{(2)} W^{\nu} \partial^{\mu} W_{\mu \nu}^{\dagger} h+\text { H.c. }\right] \\
& -\frac{1}{4 \Lambda} \tilde{g}_{h z z} Z_{\mu \nu} \tilde{Z}^{\mu \nu} h-\frac{1}{2 \Lambda} \tilde{g}_{h w w} W^{\mu \nu} \tilde{W}_{\mu \nu}^{\dagger} h .
\end{aligned}
$$

The energy scale associated with this Lagrangian is denoted by $\Lambda$; in what follows we will set $\Lambda$ to $1 \mathrm{TeV}$ for definiteness. Parametrically, modifications of the
Standard Model predictions due to operators in Eq. (5) are controlled by the quantities $g_{h V V}^{(i)} v / \Lambda$ where $v=$ $246 \mathrm{GeV}$ is the Higgs field vacuum expectation value. In what follows, we will consider values of the couplings that lead to relatively small deviations from Standard Model predictions and discuss to what extent better quality theoretical predictions for the associated production processes $p p \rightarrow V H$ may help with detecting and analyzing such scenarios.

It is straightforward to incorporate effects of the anomalous couplings into theoretical predictions for cross sections and kinematic distributions. To this end, we note that the above Lagrangian leads to the following $H V\left(q_{1}\right) V\left(q_{2}\right)$ interaction vertex 

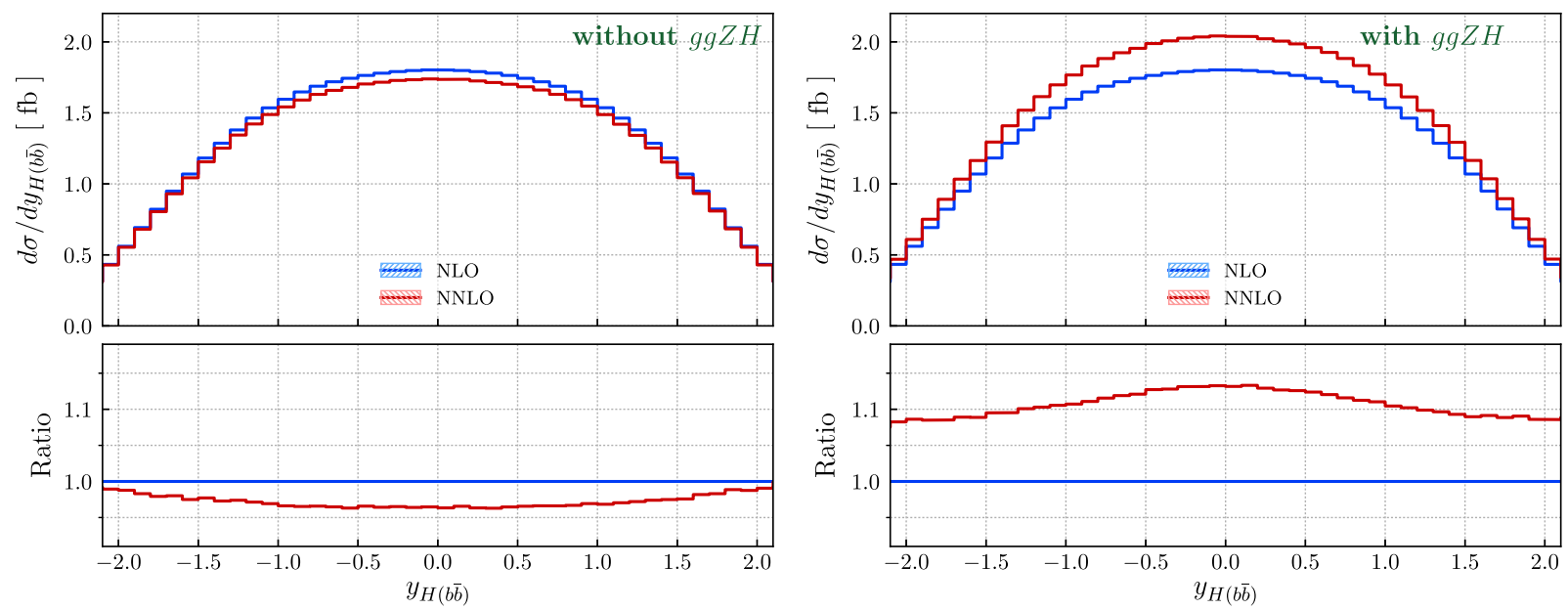

FIG. 3. The rapidity distribution of the reconstructed Higgs boson at NLO (blue) and NNLO (red) at the 13 TeV LHC with the fiducial cuts discussed in the text. We present the NNLO results without (left) and with (right) the $g g \rightarrow Z H$ contribution. We display results for the central scale $\mu=\frac{1}{2} \sqrt{\left(p_{V}+p_{H}\right)^{2}}$. The lower panes show the ratio of the NNLO results to the NLO ones. See text for details.
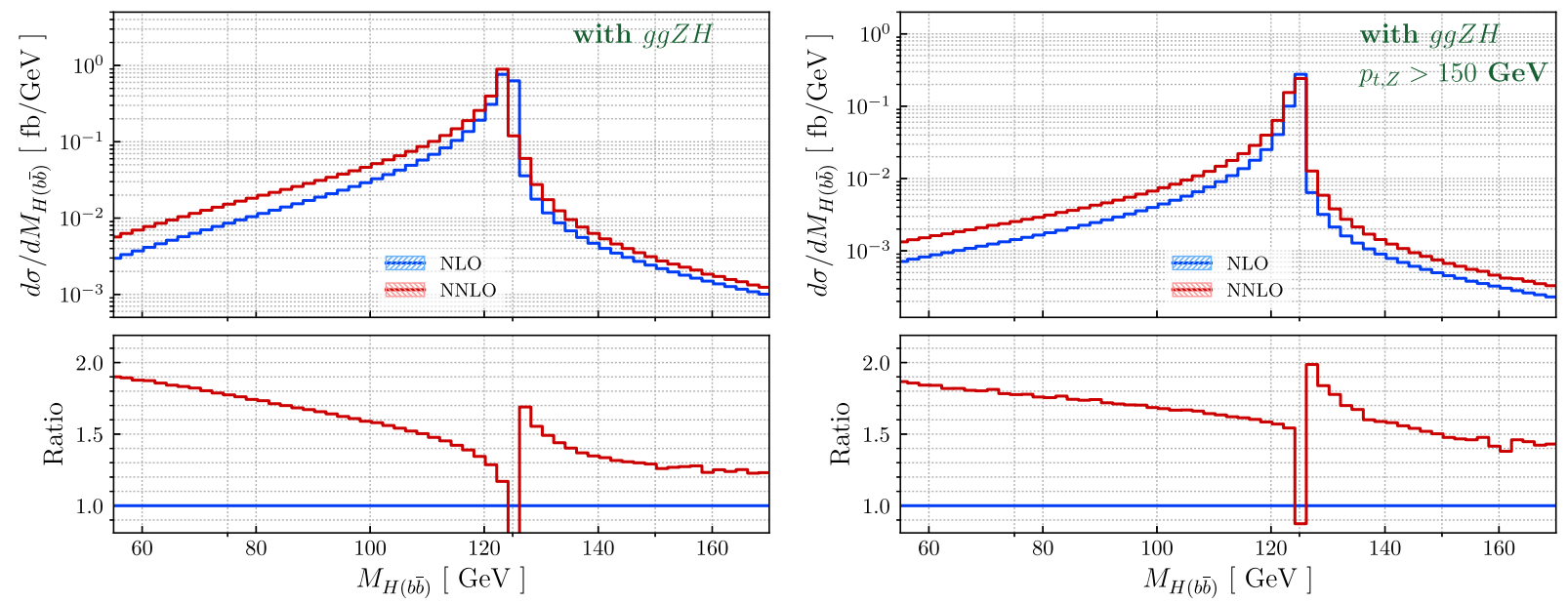

FIG. 4. The invariant mass distribution of the reconstructed Higgs boson at NLO (blue) and NNLO (red) at the 13 TeV LHC. We present the NNLO results including the $g g \rightarrow Z H$ contribution. The left plot includes the standard fiducial cuts described in the text, the right plot includes the additional $p_{t, V}>150 \mathrm{GeV}$ cut. We display results for the central scale $\mu=\frac{1}{2} \sqrt{\left(p_{V}+p_{H}\right)^{2}}$. The lower panes show the ratio of the NNLO results to the NLO ones. See text for details.

$$
\begin{aligned}
- & g^{\mu \nu} c_{1}+c_{2}\left(q_{1}^{\mu} q_{2}^{\nu}+q_{1}^{\nu} q_{2}^{\mu}\right)+c_{3} \epsilon^{\mu \nu \alpha \beta} q_{1, \alpha} q_{2, \beta} \\
& +c_{4}\left(q_{1}^{\mu} q_{1}^{\nu}+q_{2}^{\mu} q_{2}^{\nu}\right) .
\end{aligned}
$$

In Eq. (6) the coefficient $c_{1}$ is a first-degree polynomial in $q_{1}^{2}, q_{2}^{2}$ and $q_{1} q_{2}$, whereas coefficients $c_{2,3,4}$ are independent of the external momenta. Also, the coefficients $c_{1, \ldots, 4}$ are functions of the various couplings $g_{h V V}$ in the Lagrangian Eq. (5); the exact relations between the coefficients $c_{1 . .4}$ and the various $g_{h V V}$ couplings can be found in Fig. 1 of Ref. [73] and we do not reproduce them here.

We note that there has been tremendous progress in the study of EFTs for the Standard Model. In particular, if one is only interested in three-point interactions, it is possible to obtain an exact closed-form expressions to all orders in $v / \Lambda$ [85]. However, as we mentioned previously, in this paper we are primarily interested in the interplay between radiative corrections and BSM effects that could modify the shapes of distributions, rather than on the subtleties of characterizing such BSM effects. We therefore defer the NNLO-accurate study of anomalous Higgs couplings using a more suitable EFT parametrization to future work.

As we already mentioned in Sec. II, analytic formulas for the integrated subtraction terms required for the NNLO QCD description of color-singlet production [75] are generic. For this reason all that we need to do in order to incorporate effects of the anomalous couplings into a NNLO QCD description of the $p p \rightarrow V H$ process is to 
provide scattering amplitudes for hard processes $q \bar{q}^{\prime} \rightarrow$ $H l_{1} \bar{l}_{2}, q \bar{q}^{\prime} \rightarrow H l_{1} \bar{l}_{2}+g, q \bar{q}^{\prime} \rightarrow H l_{1} \bar{l}_{2}+g g$ etc. that include the anomalous couplings. Once these amplitudes are available - and it is quite straightforward to calculate them-they can be immediately included in a numerical code for computing cross sections and kinematic distributions for the associated production processes through NNLO in perturbative QCD.

In general, modifying the $H V V$ couplings will change the Higgs width from its SM value. However, in the results presented in this paper, we will keep the Higgs width fixed at its $\mathrm{SM}$ value $\Gamma_{H}=4.165 \mathrm{MeV}$. There are two reasons for doing so. First, on-shell measurements of the Higgs couplings cannot disentangle the couplings and the width, and can only extract the ratio of the couplings and the width. Second, we are mainly interested in the interplay between the effects of anomalous couplings and those arising from NNLO corrections. As the Higgs width appears as an overall rescaling factor, it does not impact either the $k$-factors or the shape changes due to these corrections. We therefore stress that, if one were to use our results in this section as absolute predictions, one should rescale the numbers to account for the modified Higgs decay width.

It is to be expected that generic choices of anomalous couplings would lead to significant changes in cross sections and kinematic distributions. For such cases an extraction of the values of the anomalous couplings from data, rather than the detection of anomalies, would benefit from precise predictions for observables that include the anomalous couplings. On the other hand, there are also cases where, even with anomalous couplings, changes in cross sections are marginal. In this situation, studies of kinematic distributions and precise theoretical predictions may be needed to both detect the presence of anomalies and distinguish between different scenarios. In what follows we present a few examples.

We have seen in the previous section that existing predictions for the $g g \rightarrow Z H$ partonic process are insufficiently precise, leaving up to $6 \%$ uncertainty in predictions for fiducial cross sections. Although this channel is sensitive to the couplings of the Higgs $[44,81,82,84]$, the fact that it is not well controlled theoretically means that it is not ideal for the kind of precision analysis that we are performing. For this reason, it is desirable to reduce the impact of this contribution. Since the relevance of the $g g \rightarrow$ $\mathrm{ZH}$ channel grows at high invariant masses of the $\mathrm{ZH}$ system, putting an upper kinematic cut on $M_{Z H}$ is useful to increase the quality of theoretical predictions without reducing fiducial cross sections. At the same time, an upper kinematic cut on $M_{Z H}$ has the additional benefit of removing contributions of high-energy tails of distributions where an EFT expansion may become unreliable. We note that since it is not possible to impose such a cut on the $W^{+} H$ system, we also restrict the transverse momentum of the vector boson following the experimental analysis [86].
Hence, we will study fiducial cross sections and kinematic distributions of the associated production processes $p p \rightarrow V H$ including the anomalous couplings by imposing the kinematic cuts of Eq. (4) as well as the following constraints:

$$
\begin{gathered}
Z H: 75 \mathrm{GeV}<p_{t, Z}<250 \mathrm{GeV}, \quad M_{e^{+} e^{-} b \bar{b}}<320 \mathrm{GeV}, \\
W^{+} H: 150 \mathrm{GeV}<p_{t, W}<250 \mathrm{GeV}
\end{gathered}
$$

The notation $M_{e^{+} e^{-} b \bar{b}}$ emphasizes the fact that the invariant mass of the $\mathrm{ZH}$ system is calculated using the fourmomenta of the two charged leptons and the two $b$ jets used for the Higgs boson reconstruction.

\section{A. $Z H$ process}

We begin with the discussion of the $p p \rightarrow Z H$ process and consider the following four scenarios ${ }^{3}$ :

$\begin{array}{llll}\text { Setup 1: } & g_{h z z}^{(1)}=+2.80, & g_{h z z}^{(2)}=-0.60, & \tilde{g}_{h z z}=+0.00, \\ \text { Setup 2: } & g_{h z z}^{(1)}=+1.05, & g_{h z z}^{(2)}=+0.00, & \tilde{g}_{h z z}=-2.90, \\ \text { Setup 3: } & g_{h z z}^{(1)}=+0.00, & g_{h z z}^{(2)}=-1.00, & \tilde{g}_{h z z}=-3.30, \\ \text { Setup 4: } & g_{h z z}^{(1)}=+1.00, & g_{h z z}^{(2)}=-1.00, & \tilde{g}_{h z z}=+2.00 .\end{array}$

Although these choices look quite random, the corresponding scenarios were chosen to provide almost identical cross sections both at leading and, especially, at next-toleading order in QCD, subject to the kinematic constraints shown in Eq. (4) and Eq. (7). This can be clearly seen from the results for fiducial cross sections summarized in Table III. We observe that NNLO QCD corrections in these cases are not insignificant; they lead to important shifts compared to next-to-leading order predictions. Also, the very strong degeneracy of the four scenarios at NLO is lifted at NNLO. However, the differences between predictions for the different scenarios remain within a few percent of each other, making NNLO QCD precision for these cases essential. It also follows from Table III that for such situations it is important to include higher-order QCD corrections to the description of processes with anomalous couplings since simply reweighting the leading order predictions with Standard Model $K$-factors may be insufficient. At any rate, having high-precision predictions for fiducial cross sections of processes with anomalous couplings may help with analyzing cases where differences between various scenarios are marginal.

Another way to lift the degeneracies of different scenarios is to explore kinematic distributions. In many cases

\footnotetext{
${ }^{3}$ We emphasize that the anomalous couplings affect both the DY-like $q \bar{q} \rightarrow Z H+X$ channels and the $g g \rightarrow Z H$ channel, the latter through their impact on triangle diagrams with a $Z Z H$ vertex.
} 
TABLE III. Fiducial cross sections for $p p \rightarrow Z H \rightarrow\left(e^{-} e^{+}\right)(b \bar{b})$ at the $13 \mathrm{TeV}$ LHC at various orders of QCD perturbation theory calculated with massive $b$ quarks. We show the results for various scenarios including anomalous couplings. We set the factorization and renormalization scales equal to each other, $\mu_{r}=\mu_{f}=\mu$. We use $\mu=\frac{1}{2} \sqrt{\left(p_{V}+p_{H}\right)^{2}}$ for the central value and the uncertainties are calculated by varying the scale $\mu$ by a factor of two in both directions. See text for details.

\begin{tabular}{lccccc}
\hline \hline$\sigma_{\text {fid }}^{Z H}(\mathrm{fb})$ & SM & Setup 1 & Setup 2 & Setup 3 & Setup 4 \\
\hline LO & $0.894_{-0.041}^{+0.032}$ & $0.782_{-0.028}^{+0.028}$ & $0.854_{-0.038}^{+0.031}$ & $0.786_{-0.034}^{+0.027}$ & $0.780_{-0.034}^{+0.027}$ \\
NLO & $1.289_{-0.017}^{+0.025}$ & $1.266_{-0.007}^{+0.012}$ & $1.273_{-0.010}^{+0.011}$ & $1.276_{-0.004}^{+0.009}$ & $1.269_{-0.003}^{+0.008}$ \\
NNLO & $1.356_{-0.009}^{+0.0011}$ & $1.423_{-0.003}^{+0.003}$ & $1.379_{-0.004}^{+0.004}$ & $1.454_{-0.003}^{+0.003}$ & $1.445_{-0.004}^{+0.003}$ \\
NNLO $(+g g Z H)$ & $1.419_{-0.023}^{+0.024}$ & $1.476_{-0.015}^{+0.015}$ & $1.443_{-0.015}^{+0.028}$ & $1.499_{-0.015}^{+0.014}$ & $1.490_{-0.011}^{+0.014}$ \\
\hline \hline
\end{tabular}

kinematic distributions offer more opportunities to detect the anomalous couplings since their effects can be quite profound even if they are small in fiducial cross sections. However, for the four scenarios that we considered, the situation is slightly more subtle, as we will illustrate now.

In Fig. 5 we show distributions of the Higgs boson transverse momentum, the transverse momentum of the hardest $b$ jet, the angular separation between the hardest $b$ jet and the hardest lepton $\Delta R_{b \ell}$, and the transverse momentum distribution of the hardest lepton. The quantity $\Delta R_{b \ell}$ is defined as

$$
\Delta R_{b \ell}=\sqrt{\left(y_{b}-y_{\ell}\right)^{2}+\left(\varphi_{b}-\varphi_{\ell}\right)^{2}},
$$

where $y_{b}\left(y_{\ell}\right)$ and $\varphi_{b}\left(\varphi_{\ell}\right)$ are the rapidity and the azimuthal angle of the hardest $b$ jet (the hardest lepton), respectively.
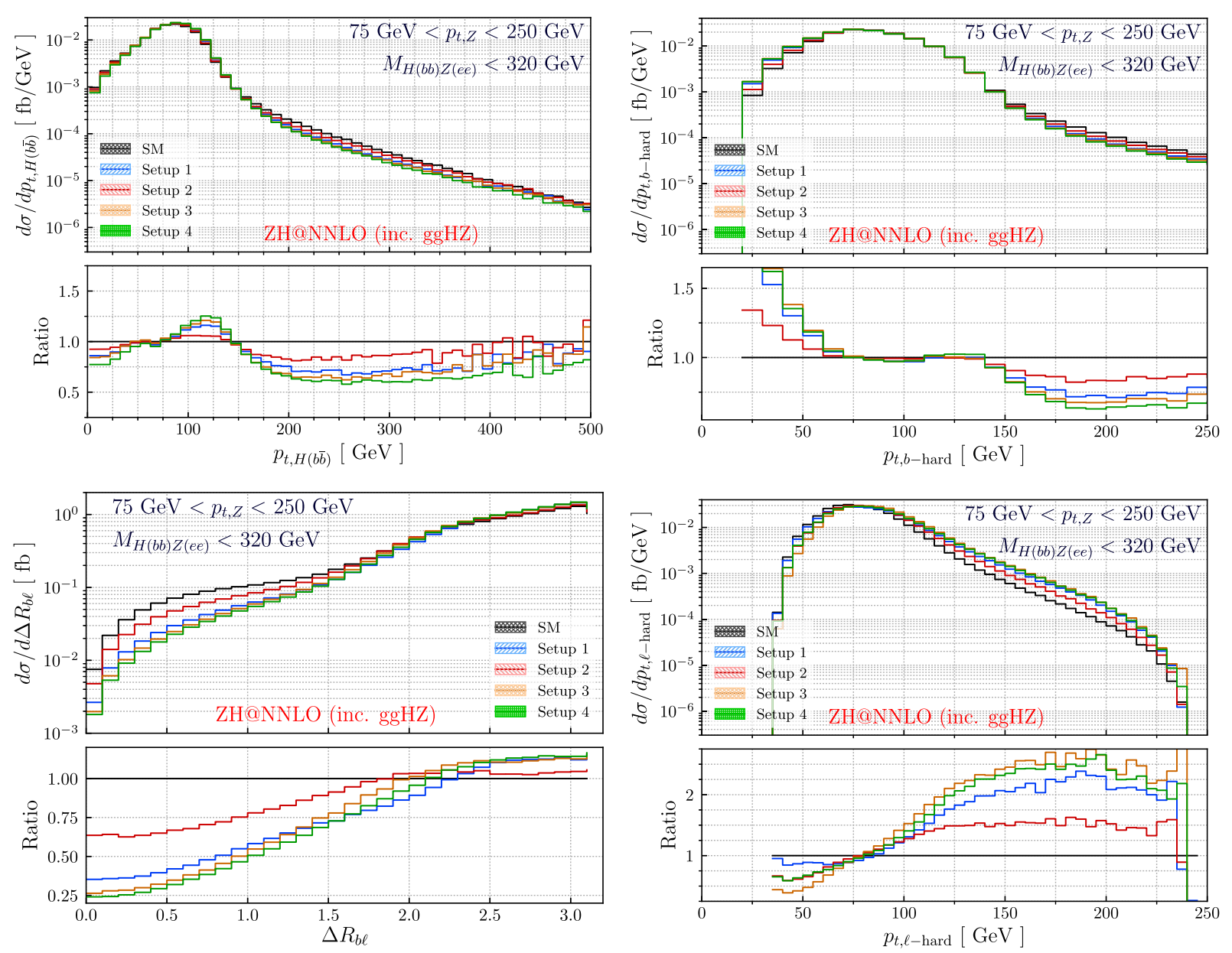

FIG. 5. Kinematic distributions in the process $p p \rightarrow Z\left(e^{+} e^{-}\right) H(b \bar{b})$ at the $13 \mathrm{TeV}$ LHC for various SMEFT scenarios. In the lower panes, ratios of SMEFT to SM distributions are shown. We set the factorization and the renormalization scales in the production process to half the invariant mass of the $Z H$ system. See text for details. 
TABLE IV. Fiducial cross sections for $p p \rightarrow W^{+} H \rightarrow\left(\nu_{e} e^{+}\right)(b \bar{b})$ at the $13 \mathrm{TeV}$ LHC at various orders of QCD perturbation theory calculated with massive $b$ quarks. We show the results for various scenarios including anomalous couplings. We set the factorization and renormalization scales equal to each other, $\mu_{r}=\mu_{f}=\mu$. We use $\mu=\frac{1}{2} \sqrt{\left(p_{V}+p_{H}\right)^{2}}$ for the central value and the uncertainties are calculated by varying the scale $\mu$ by a factor of two in both directions. See main text for details.

\begin{tabular}{lcccrr}
\hline \hline$\sigma_{\text {fid }}^{W^{+} H}(\mathrm{fb})$ & SM & Setup 1 & Setup 2 & Setup 3 & Setup 4 \\
\hline LO & $2.813_{-0.039}^{+0.023}$ & $2.657_{-0.024}^{+0.012}$ & $2.999_{-0.021}^{+0.007}$ & $2.898_{-0.026}^{+0.012}$ & $2.958_{-0.021}^{+0.007}$ \\
NLO & $3.434_{-0.064}^{+0.089}$ & $3.419_{-0.080}^{+0.110}$ & $3.466_{-0.048}^{+0.070}$ & $3.501_{-0.063}^{+0.088}$ & $3.458_{-0.052}^{+0.074}$ \\
NNLO & $3.409_{-0.025}^{+0.024}$ & $3.436_{-0.034}^{+0.028}$ & $3.387_{-0.015}^{+0.004}$ & $3.463_{-0.031}^{+0.015}$ & $3.390_{-0.018}^{+0.003}$ \\
\hline \hline
\end{tabular}

It follows from Fig. 5 that there are kinematic regions where the differences between the four scenarios are more pronounced than in fiducial cross sections. For example, if we look at the $p_{t, H(b \bar{b})}$ distribution, there are noticeable differences at low transverse momenta, whereas in the peak region all four distributions are similar. The same applies to the other three distributions. For example, the $\Delta R_{b l}$ distributions peak at $\Delta R_{b l} \sim 3$; in that region the four scenarios provide very similar results. The differences become noticeable at $\Delta R_{b l} \sim 1$ but the number of events
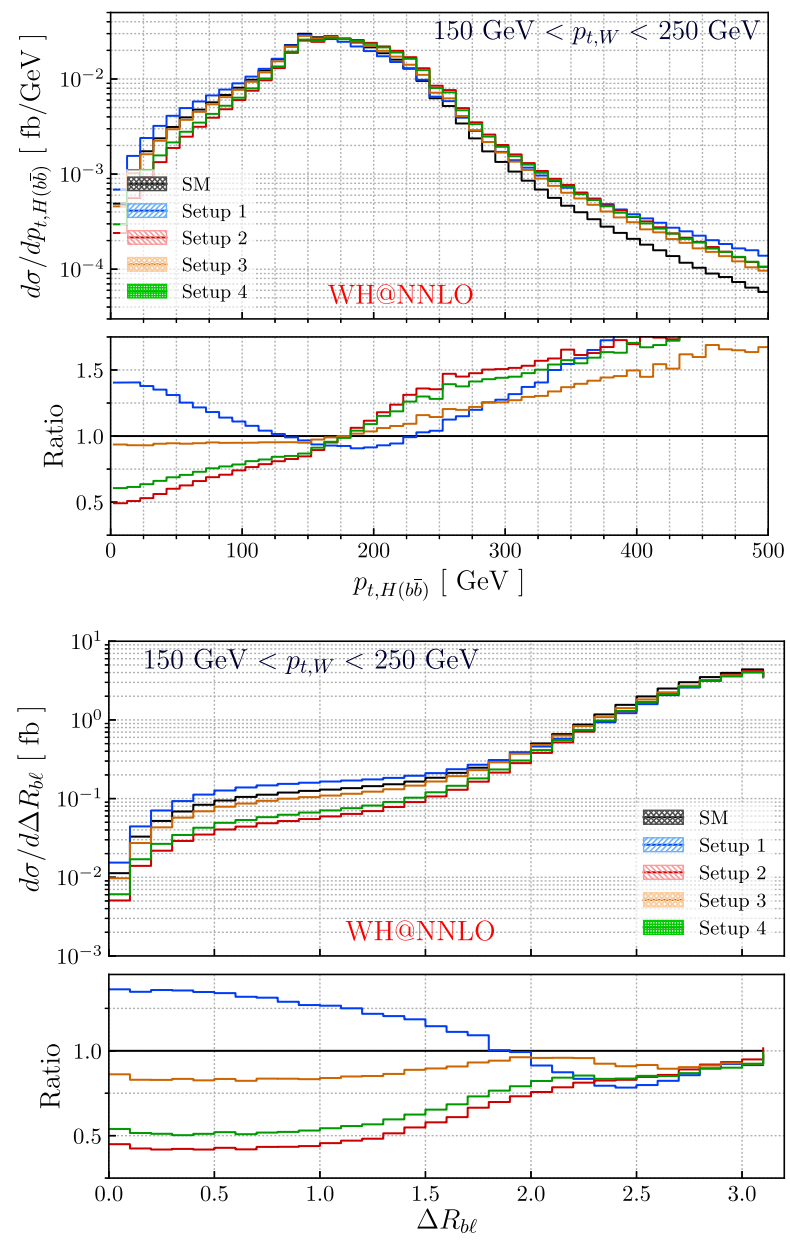

for such values of $\Delta R_{b l}$ is reduced by an order of magnitude. Given the fact that we deal here with $\mathcal{O}(1 \mathrm{fb})$ cross sections, losing an order of magnitude in the number of events is not optimal. However, the availability of highly accurate NNLO QCD predictions in peak regions of kinematic distributions and identifiable differences between various scenarios in distribution tails should allow one to optimize analysis strategies and benefit from measurements across accessible kinematic regions.
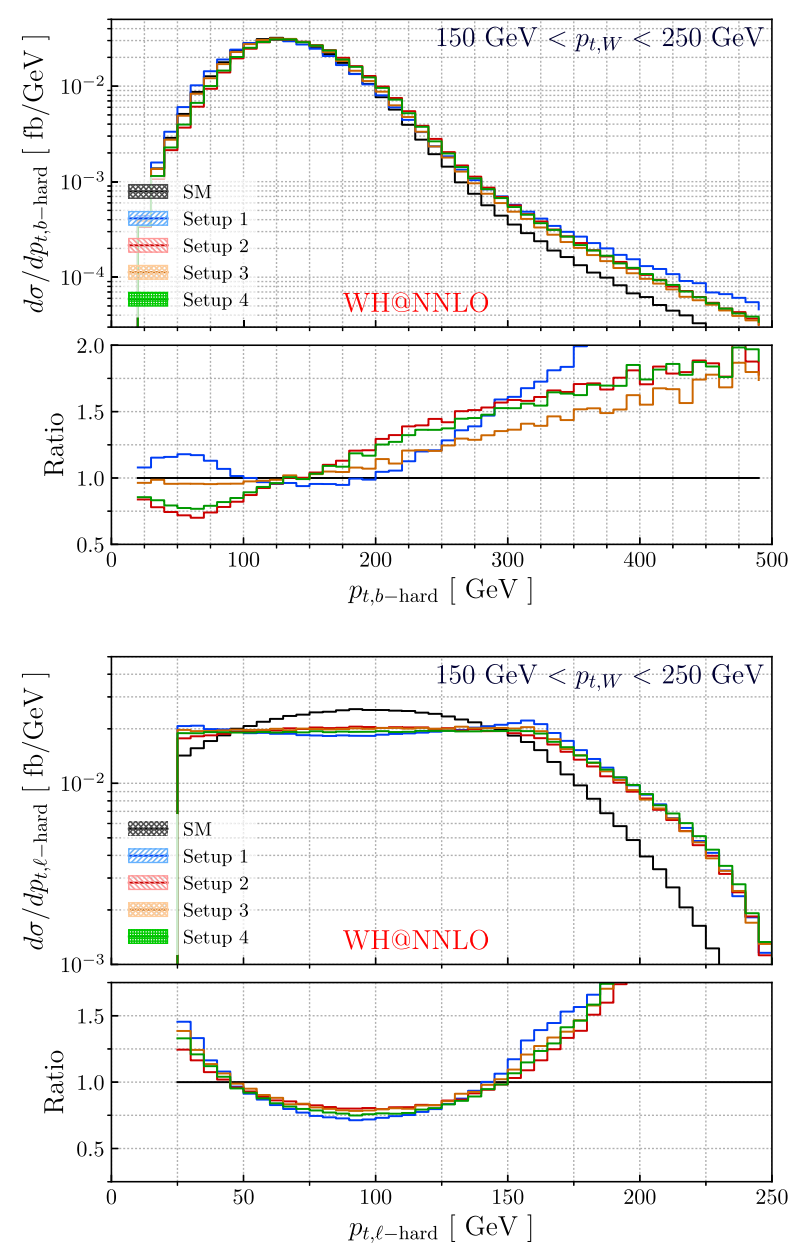

FIG. 6. Kinematic distributions in the process $p p \rightarrow W^{+}\left(e^{+} \nu\right) H(b \bar{b})$ at the $13 \mathrm{TeV}$ LHC for various SMEFT scenarios. In lower panes ratios of SMEFT to SM distributions are shown. We set the factorization and the renormalization scales in the production process to half the invariant mass of the $W H$ system. See text for details. 


\section{B. $W^{+} \boldsymbol{H}$ process}

We repeat the analysis of the previous subsection for $W^{+} H$ production. We focus exclusively on the fiducial region defined in Eq. (4) with additional restrictions on the $W$-boson transverse momentum, shown in Eq. (8).

We consider four different scenarios of the anomalous couplings and we choose them in a way that makes the differences between fiducial cross sections marginal. The four scenarios are:

Setup 1: $\quad g_{h w w}^{(1)}=-1.20, \quad g_{h w w}^{(2)}=-0.25, \quad \tilde{g}_{h w w}=+0.00$,

Setup 2: $\quad g_{h w w}^{(1)}=+1.00, \quad g_{h w w}^{(2)}=+0.00, \quad \tilde{g}_{h w w}=+0.80$,

Setup 3: $\quad g_{h w w}^{(1)}=+0.00, \quad g_{h w w}^{(2)}=-0.10, \quad \tilde{g}_{h w w}=-1.10$,

Setup 4: $\quad g_{h w w}^{(1)}=+0.70, \quad g_{h w w}^{(2)}=-0.05, \quad \tilde{g}_{h w w}=-1.05$.

The fiducial cross sections at various orders of perturbation theory are reported in Table IV. We observe that the NLO QCD predictions for cross sections for the four scenarios agree to within a few percent. At variance with $\mathrm{ZH}$ case, however, adding NNLO QCD corrections does not change the situation in a significant way except that the uncertainty on the theoretical predictions is reduced compared to the NLO QCD case. However, we again observe that the NNLO QCD corrections are not constant across the four scenarios.

In Fig. 6 we show kinematic distributions for the $p p \rightarrow$ $W^{+} H$ process for the four scenarios considered above. Overall, the situation is similar to what has been already discussed in case of $p p \rightarrow Z H$ : in peak regions of all distributions the different scenarios provide very similar predictions; away from peak regions clear differences are seen in some of them. These differences, as well as reduced theoretical uncertainties in peak regions, should eventually enable improved studies of the anomalous couplings in $W^{+} H$ production.

Before concluding we would like to illustrate the potential impact of the calculations described in this paper on bounds on the anomalous couplings that can be obtained from measurements of fiducial cross sections of $p p \rightarrow V H$ processes. We consider a hypothetical measurement of a fiducial $W^{+} H$ cross section and find the allowed values for various combinations of the anomalous couplings. We use the same setup as described earlier in this section to define the fiducial $W^{+} H$ cross section. We assume that it has been measured and the value $\sigma_{\text {fidexp }}^{W^{+} H}=3.40(14) \mathrm{fb}$ was obtained. We assigned a $4 \%$ uncertainty to the measured cross section; this corresponds to projections for the high-luminosity LHC that can be found in Ref. [87].
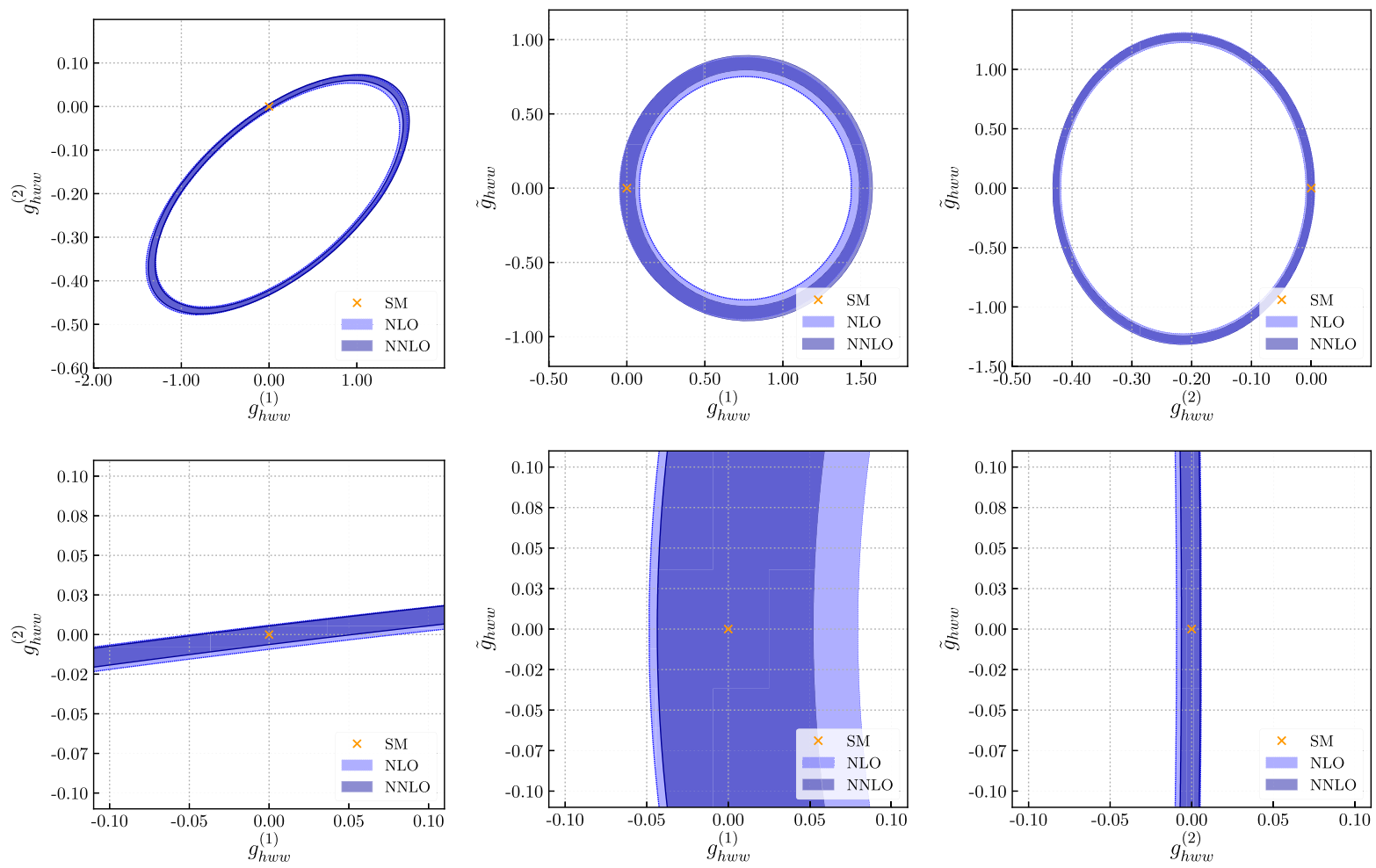

FIG. 7. Examples of contours ( $68 \%$ confidence level) of allowed combinations of anomalous couplings based on a hypothetical measurement of the fiducial cross section of $W^{+} H$ production at the $13 \mathrm{TeV}$ LHC. Color coding describes NLO (lighter blue) and NNLO (darker blue) calculations. The SM result is shown as an orange cross. Upper row: full contours of allowed couplings; lower row: contours close to the SM configuration, i.e., for small anomalous couplings. A $4 \%$ experimental uncertainty was assumed. See text for details. 

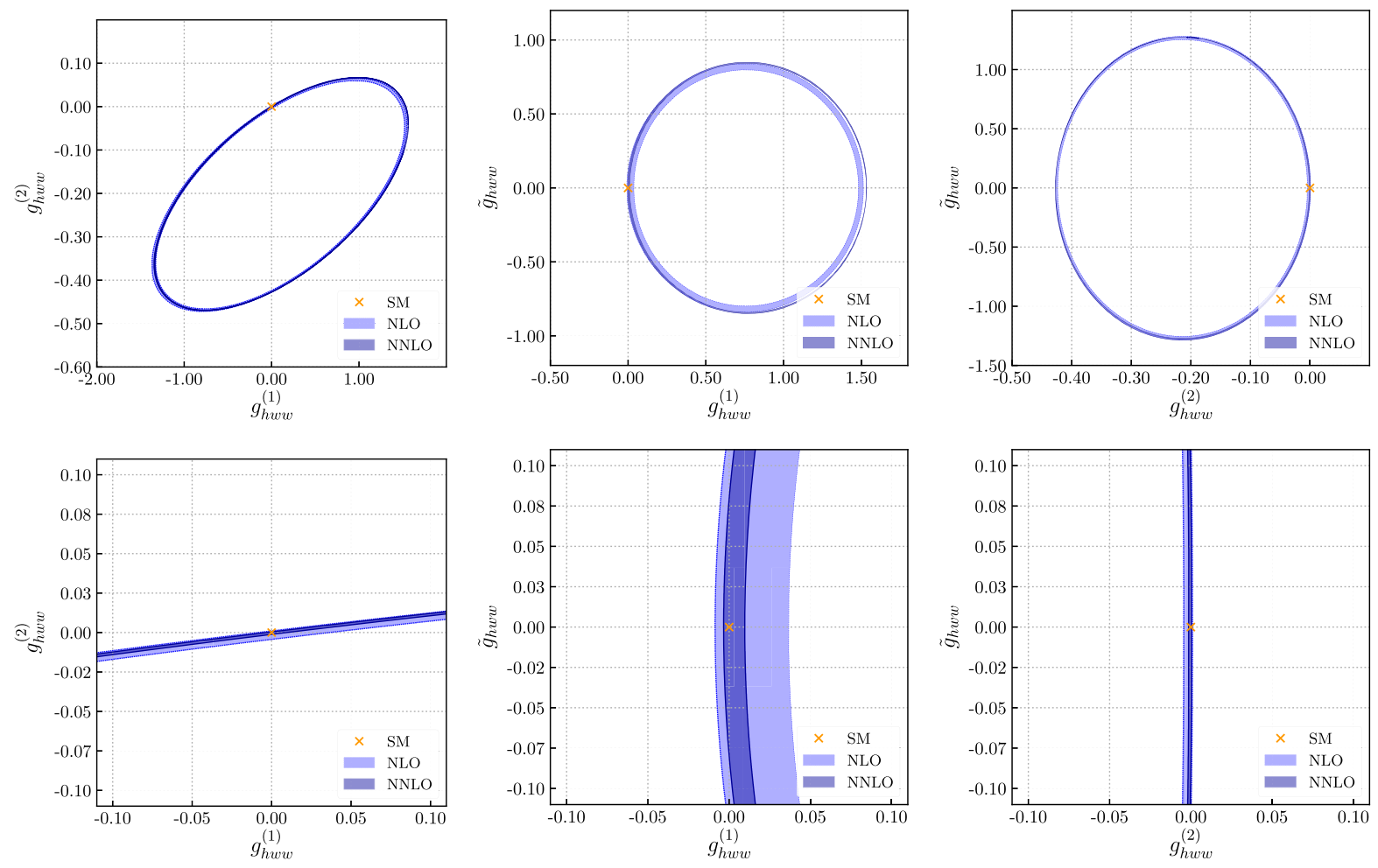

FIG. 8. As for Fig. 7 but with the experimental uncertainty removed. See text for details.

We would like to understand how regions of allowed anomalous couplings change when we increase the accuracy of theoretical computations. We note that the fiducial cross section $\sigma_{\text {fid }}^{W H}=f\left(g_{h w w}^{(1)}, g_{h w w}^{(2)}, \tilde{g}_{h w w}\right)$ is a polynomial in the couplings. For this reason, it is enough to sample it for ten different points to determine the full function. We then use these results to check which combinations of anomalous couplings are compatible with the result of the hypothetical measurement. The uncertainty in the experimental cross section is fixed to $4 \%$ and the uncertainties in the theoretical predictions is determined by varying the scale by a factor of two around the central scale $\mu=$ $M_{W H} / 2$. We note that the factorization and the renormalization scales are chosen to be equal.

Our results are presented in Fig. 7 where we show twodimensional projections of the $g_{h w w}^{(i)}$ parameter space. Shaded areas mark couplings that are compatible with the results of the measurement at the $68 \%$ confidence level. We note that NLO QCD corrections change the LO predictions significantly; for this reason, we do not display the latter. Changes are smaller when moving from NLO to NNLO predictions; nevertheless, we observe some distortion of shapes of the allowed region. This effect is a consequence of the fact that corrections do, in fact, depend on the anomalous couplings, a feature that we have already discussed when talking about Table IV.

The thickness of the bands representing the allowed regions is only marginally reduced when NNLO predictions are used instead of NLO predictions. This is a consequence of the fact that the experimental uncertainty is fixed at $4 \%$ which is comparable to the scale uncertainty of the theoretical prediction at NLO and is larger than that of the NNLO prediction.

In order to highlight potential benefits of using NNLO theory predictions, we display similar exclusion limits in Fig. 8 but assume that the experimental uncertainty is significantly reduced. For the sake of argument, we take it to be zero. We can now clearly see the thickness of the bands decreasing as we move from NLO to NNLO, as a result of the decreased theoretical error. Of course, it is unrealistic to assume no experimental uncertainty, but using this assumption does allow us to highlight the benefits of NNLO-accurate theoretical predictions. We note, in this regard, that projections in Ref. [87] are only estimates and that it is quite possible that the actual results will outperform these projections. If this happens, we anticipate that fully differential NNLO theoretical predictions will become not only preferable but perhaps even necessary for studies of the anomalous couplings in the $p p \rightarrow V H$ process.

\section{CONCLUSIONS}

In this paper, we presented computations of NNLO QCD corrections to Higgs boson production in association with a $W$ or $Z$ boson. We included NNLO QCD corrections to $H \rightarrow b \bar{b}$ decays, retaining full $b$-quark mass dependence. This allowed us to present our results in a setup which is close to the actual experimental analyses. 
In addition to NNLO QCD corrections and the effects of the $b$-quark masses, our computation also includes anomalous couplings in the $V V H$ interaction vertex. We have shown that QCD corrections to fiducial cross sections depend nontrivially on the anomalous couplings since they change the relative importance of various kinematic regions that contribute to the fiducial cross sections. We have argued that the availability of NNLO QCD predictions may allow one to search for the anomalous couplings in kinematic regions where the EFT framework based on the momentum expansion is more trustworthy than in the high-energy tails of distributions. We have also shown how the NNLO QCD theory predictions may be used to improve exclusion limits for the anomalous couplings; this becomes especially relevant if experimental uncertainties on fiducial cross sections of $p p \rightarrow V H$ production measured at the high-luminosity LHC reach the few percent level.

The computation reported in this paper describes the most advanced and realistic way to simulate the associated production process $p p \rightarrow V H$ at the LHC, but further improvements are possible. On the SM side, it is definitely important to refine the calculation of the $g g \rightarrow \mathrm{ZH}$ subprocess and to update the contributions to $\mathrm{VH}$ production that depend on top loops since many of them are still only known as expansions in the inverse mass of the top quark.

On the EFT side, it would be interesting to repeat this analysis using the exact EFT parametrization of Ref. [85]. One can also start including contributions of other dimension-six operators, gradually moving toward a full EFT analysis. Although some of these extensions of the current computation are not trivial, they are clearly possible given recent developments in both methods for loop computations and subtraction technology. We look forward to providing such refined predictions for $V H$ associated production in the future.

\section{ACKNOWLEDGMENTS}

This research is partially supported by the Deutsche Forschungsgemeinschaft (DFG, German Research Foundation) under grant No. 396021762-TRR 257. The research of F.C. was partially supported by the ERC Starting Grant No. 804394 hipQCD.
[1] G. Aad et al. (ATLAS and CMS Collaborations), Measurements of the Higgs boson production and decay rates and constraints on its couplings from a combined ATLAS and CMS analysis of the LHC pp collision data at $\sqrt{s}=7$ and $8 \mathrm{TeV}$, J. High Energy Phys. 08 (2016) 045.

[2] J. M. Butterworth, A. R. Davison, M. Rubin, and G. P. Salam, Jet Substructure as a New Higgs Search Channel at the LHC, Phys. Rev. Lett. 100, 242001 (2008).

[3] M. Aaboud et al. (ATLAS Collaboration), Evidence for the $H \rightarrow b \bar{b}$ decay with the ATLAS detector, J. High Energy Phys. 12 (2017) 024.

[4] A. M. Sirunyan et al. (CMS Collaboration), Evidence for the Higgs boson decay to a bottom quark-antiquark pair, Phys. Lett. B 780, 501 (2018).

[5] M. Aaboud et al. (ATLAS Collaboration), Observation of $H \rightarrow b \bar{b}$ decays and $V H$ production with the ATLAS detector, Phys. Lett. B 786, 59 (2018).

[6] A. M. Sirunyan et al. (CMS Collaboration), Observation of Higgs Boson Decay to Bottom Quarks, Phys. Rev. Lett. 121, 121801 (2018).

[7] A. Behring, W. Bizoń, F. Caola, K. Melnikov, and R. Röntsch, Bottom quark mass effects in associated $W H$ production with the $H \rightarrow b \bar{b}$ decay through NNLO QCD, Phys. Rev. D 101, 114012 (2020).

[8] T. Han and S. Willenbrock, QCD correction to the $p p \rightarrow W H$ and $Z H$ total cross-sections, Phys. Lett. B 273, 167 (1991).

[9] H. Baer, B. Bailey, and J. F. Owens, $\mathcal{O}\left(\alpha_{s}\right)$ Monte Carlo approach to $W+$ Higgs associated production at hadron supercolliders, Phys. Rev. D 47, 2730 (1993).
[10] J. Ohnemus and W. J. Stirling, Order $\alpha_{s}$ corrections to the differential cross-section for the $W H$ intermediate mass Higgs signal, Phys. Rev. D 47, 2722 (1993).

[11] S. Mrenna and C. P. Yuan, Effects of QCD resummation on $\mathrm{W}^{+} h$ and $t \bar{b}$ production at the Tevatron, Phys. Lett. B 416, 200 (1998).

[12] M. Spira, QCD effects in Higgs physics, Fortschr. Phys. 46, 203 (1998).

[13] A. Djouadi and M. Spira, SUSY-QCD corrections to Higgs boson production at hadron colliders, Phys. Rev. D 62, 014004 (2000).

[14] O. Brein, A. Djouadi, and R. Harlander, NNLO QCD corrections to the Higgs-strahlung processes at hadron colliders, Phys. Lett. B 579, 149 (2004).

[15] O. Brein, R. Harlander, M. Wiesemann, and T. Zirke, Top-quark mediated effects in hadronic Higgs-Strahlung, Eur. Phys. J. C 72, 1868 (2012).

[16] O. Brein, R. V. Harlander, and T. J. E. Zirke, vh@nnloHiggs-Strahlung at hadron colliders, Comput. Phys. Commun. 184, 998 (2013).

[17] G. Ferrera, M. Grazzini, and F. Tramontano, Associated WH Production at Hadron Colliders: A Fully Exclusive QCD Calculation at NNLO, Phys. Rev. Lett. 107, 152003 (2011).

[18] G. Ferrera, M. Grazzini, and F. Tramontano, Higher-order QCD effects for associated WH production and decay at the LHC, J. High Energy Phys. 04 (2014) 039.

[19] G. Ferrera, M. Grazzini, and F. Tramontano, Associated ZH production at hadron colliders: The fully differential NNLO QCD calculation, Phys. Lett. B 740, 51 (2015). 
[20] J. M. Campbell, R. K. Ellis, and C. Williams, Associated production of a Higgs boson at NNLO, J. High Energy Phys. 06 (2016) 179.

[21] G. Ferrera, G. Somogyi, and F. Tramontano, Associated production of a Higgs boson decaying into bottom quarks at the LHC in full NNLO QCD, Phys. Lett. B 780, 346 (2018).

[22] F. Caola, G. Luisoni, K. Melnikov, and R. Röntsch, NNLO QCD corrections to associated $W H$ production and $H \rightarrow b \bar{b}$ decay, Phys. Rev. D 97, 074022 (2018).

[23] R. Gauld, A. Gehrmann-De Ridder, E. W. N. Glover, A. Huss, and I. Majer, Associated production of a Higgs boson decaying into bottom quarks and a weak vector boson decaying leptonically at NNLO in QCD, J. High Energy Phys. 10 (2019) 002.

[24] M. L. Ciccolini, S. Dittmaier, and M. Kramer, Electroweak radiative corrections to associated $\mathrm{WH}$ and $\mathrm{ZH}$ production at hadron colliders, Phys. Rev. D 68, 073003 (2003).

[25] A. Denner, S. Dittmaier, S. Kallweit, and A. Muck, Electroweak corrections to Higgs-Strahlung off W/Z bosons at the Tevatron and the LHC with HAWK, J. High Energy Phys. 03 (2012) 075.

[26] A. Denner, S. Dittmaier, S. Kallweit, and A. Mück, HAWK2.0: A Monte Carlo program for Higgs production in vector-boson fusion and Higgs strahlung at hadron colliders, Comput. Phys. Commun. 195, 161 (2015).

[27] R. Gauld, A. Gehrmann-De Ridder, E. W. N. Glover, A. Huss, and I. Majer, Precise predictions for $\mathrm{WH}+$ jet production at the LHC, Phys. Lett. B 817, 136335 (2021).

[28] B. Yan and C. P. Yuan, The Anomalous $Z b \bar{b}$ Couplings: From LEP to LHC, Phys. Rev. Lett. 127, 051801 (2021).

[29] B. A. Kniehl, Associated production of Higgs and $Z$ bosons from gluon fusion in hadron collisions, Phys. Rev. D 42, 2253 (1990).

[30] D. A. Dicus and C. Kao, Higgs boson $-Z^{0}$ production from gluon fusion, Phys. Rev. D 38, 1008 (1988); 42, 2412 (1990).

[31] L. Altenkamp, S. Dittmaier, R. V. Harlander, H. Rzehak, and T. J. E. Zirke, Gluon-induced Higgs-Strahlung at next-toleading order QCD, J. High Energy Phys. 02 (2013) 078.

[32] R. V. Harlander, A. Kulesza, V. Theeuwes, and T. Zirke, Soft gluon resummation for gluon-induced Higgs Strahlung, J. High Energy Phys. 11 (2014) 082.

[33] A. Hasselhuhn, T. Luthe, and M. Steinhauser, On top quark mass effects to $g g \rightarrow Z H$ at NLO, J. High Energy Phys. 01 (2017) 073.

[34] J. Davies, G. Mishima, and M. Steinhauser, Virtual corrections to $g g \rightarrow Z H$ in the high-energy and large- $m_{t}$ limits, J. High Energy Phys. 03 (2021) 034.

[35] L. Chen, G. Heinrich, S. P. Jones, M. Kerner, J. Klappert, and J. Schlenk, $\mathrm{ZH}$ production in gluon fusion: Two-loop amplitudes with full top quark mass dependence, J. High Energy Phys. 03 (2021) 125.

[36] L. Alasfar, G. Degrassi, P. P. Giardino, R. Gröber, and M. Vitti, Virtual corrections to $g g \rightarrow Z H$ via a transverse momentum expansion, J. High Energy Phys. 05 (2021) 168.

[37] R. V. Harlander, J. Klappert, C. Pandini, and A. Papaefstathiou, Exploiting the WH/ZH symmetry in the search for New Physics, Eur. Phys. J. C 78, 760 (2018).
[38] W. Astill, W. Bizon, E. Re, and G. Zanderighi, NNLOPS accurate associated HW production, J. High Energy Phys. 06 (2016) 154.

[39] S. Alioli, A. Broggio, S. Kallweit, M. A. Lim, and L. Rottoli, Higgsstrahlung at NNLL' + NNLO matched to parton showers in GENEVA, Phys. Rev. D 100, 096016 (2019).

[40] W. Astill, W. Bizoń, E. Re, and G. Zanderighi, NNLOPS accurate associated $\mathrm{HZ}$ production with $H \rightarrow b \bar{b}$ decay at NLO, J. High Energy Phys. 11 (2018) 157.

[41] W. Bizoń, E. Re, and G. Zanderighi, NNLOPS description of the $H \rightarrow b \bar{b}$ decay with MiNLO, J. High Energy Phys. 06 (2020) 006.

[42] F. Granata, J. M. Lindert, C. Oleari, and S. Pozzorini, NLO $\mathrm{QCD}+\mathrm{EW}$ predictions for $\mathrm{HV}$ and $\mathrm{HV}+$ jet production including parton-shower effects, J. High Energy Phys. 09 (2017) 012.

[43] B. Hespel, F. Maltoni, and E. Vryonidou, Higgs and $Z$ boson associated production via gluon fusion in the SM and the 2HDM, J. High Energy Phys. 06 (2015) 065.

[44] D. Goncalves, F. Krauss, S. Kuttimalai, and P. Maierhöfer, Higgs-strahlung: Merging the NLO Drell-Yan and loopinduced $0+1$ jet multiplicities, Phys. Rev. D 92, 073006 (2015).

[45] S. G. Gorishnii, A. L. Kataev, S. A. Larin, and L. R. Surguladze, Corrected three loop QCD correction to the correlator of the quark scalar currents and $\Gamma$ (Tot) $\left(H^{0} \rightarrow\right.$ hadrons), Mod. Phys. Lett. A 05, 2703 (1990).

[46] S. G. Gorishnii, A. L. Kataev, S. A. Larin, and L. R. Surguladze, Scheme dependence of the next to next-toleading QCD corrections to $\Gamma$ (tot) $\left(H^{0} \rightarrow\right.$ hadrons) and the spurious QCD infrared fixed point, Phys. Rev. D 43, 1633 (1991).

[47] A. L. Kataev and V.T. Kim, The effects of the QCD corrections to $\Gamma\left(H^{0} \rightarrow b \bar{b}\right)$, Mod. Phys. Lett. A 09, 1309 (1994).

[48] L. R. Surguladze, Quark mass effects in fermionic decays of the Higgs boson in $\mathcal{O}\left(\alpha_{s}^{2}\right)$ perturbative QCD, Phys. Lett. B 341, 60 (1994).

[49] S. A. Larin, T. van Ritbergen, and J. A. M. Vermaseren, The Large top quark mass expansion for Higgs boson decays into bottom quarks and into gluons, Phys. Lett. B 362, 134 (1995).

[50] K. G. Chetyrkin and A. Kwiatkowski, Second order QCD corrections to scalar and pseudoscalar Higgs decays into massive bottom quarks, Nucl. Phys. B461, 3 (1996).

[51] K. G. Chetyrkin, Correlator of the quark scalar currents and $\Gamma$ (tot) $\left(H \rightarrow\right.$ hadrons) at $\mathcal{O}\left(\alpha_{s}^{3}\right)$ in pQCD, Phys. Lett. B 390, 309 (1997).

[52] P. A. Baikov, K. G. Chetyrkin, and J. H. Kuhn, Scalar Correlator at $\mathcal{O}\left(\alpha_{s}^{4}\right)$, Higgs Decay into b-Quarks and Bounds on the Light Quark Masses, Phys. Rev. Lett. 96, 012003 (2006).

[53] J. Davies, M. Steinhauser, and D. Wellmann, Completing the hadronic Higgs boson decay at order $\alpha_{s}^{4}$, Nucl. Phys. B920, 20 (2017).

[54] J. Fleischer and F. Jegerlehner, Radiative corrections to Higgs decays in the extended Weinberg-Salam model, Phys. Rev. D 23, 2001 (1981). 
[55] D. Y. Bardin, B. M. Vilensky, and P. K. Khristova, Calculation of the Higgs boson decay width into fermion pairs, Sov. J. Nucl. Phys. 53, 152 (1991).

[56] A. Dabelstein and W. Hollik, Electroweak corrections to the fermionic decay width of the standard Higgs boson, Z. Phys. C 53, 507 (1992).

[57] B. A. Kniehl, Radiative corrections for $H \rightarrow f$ anti-f $(\gamma)$ in the standard model, Nucl. Phys. B376, 3 (1992).

[58] L. Mihaila, B. Schmidt, and M. Steinhauser, $\Gamma(H \rightarrow b \bar{b})$ to order $\alpha \alpha_{s}$, Phys. Lett. B 751, 442 (2015).

[59] A. Denner, S. Heinemeyer, I. Puljak, D. Rebuzzi, and M. Spira, Standard model Higgs-boson branching ratios with uncertainties, Eur. Phys. J. C 71, 1753 (2011).

[60] M. Spira, Higgs boson production and decay at hadron colliders, Prog. Part. Nucl. Phys. 95, 98 (2017).

[61] C. Anastasiou, F. Herzog, and A. Lazopoulos, The fully differential decay rate of a Higgs boson to bottom-quarks at NNLO in QCD, J. High Energy Phys. 03 (2012) 035.

[62] V. Del Duca, C. Duhr, G. Somogyi, F. Tramontano, and Z. Trócsányi, Higgs boson decay into b-quarks at NNLO accuracy, J. High Energy Phys. 04 (2015) 036.

[63] F. Caola, K. Melnikov, and R. Röntsch, Analytic results for decays of color singlets to $g g$ and $q \bar{q}$ final states at NNLO QCD with the nested soft-collinear subtraction scheme, Eur. Phys. J. C 79, 1013 (2019).

[64] R. Mondini and C. Williams, $H \rightarrow b \bar{b} j$ at next-to-next-toleading order accuracy, J. High Energy Phys. 06 (2019) 120.

[65] R. Mondini, M. Schiavi, and C. Williams, N ${ }^{3}$ LO predictions for the decay of the Higgs boson to bottom quarks, J. High Energy Phys. 06 (2019) 079.

[66] W. Bernreuther, L. Chen, and Z.-G. Si, Differential decay rates of $C P$-even and $C P$-odd Higgs bosons to top and bottom quarks at NNLO QCD, J. High Energy Phys. 07 (2018) 159.

[67] A. Behring and W. Bizoń, Higgs decay into massive b-quarks at NNLO QCD in the nested soft-collinear subtraction scheme, J. High Energy Phys. 01 (2020) 189.

[68] G. Somogyi and F. Tramontano, Fully exclusive heavy quark-antiquark pair production from a colourless initial state at NNLO in QCD, J. High Energy Phys. 11 (2020) 142.

[69] A. Primo, G. Sasso, G. Somogyi, and F. Tramontano, Exact Top Yukawa corrections to Higgs boson decay into bottom quarks, Phys. Rev. D 99, 054013 (2019).

[70] R. Mondini, U. Schubert, and C. Williams, Top-induced contributions to $H \rightarrow b \bar{b}$ and $H \rightarrow c \bar{c}$ at $\mathcal{O}\left(\alpha_{s}^{3}\right)$, J. High Energy Phys. 12 (2020) 058.

[71] I. Brivio and M. Trott, The standard model as an effective field theory, Phys. Rep. 793, 1 (2019).

[72] A. Banerjee, S. Dasgupta, and T. S. Ray, Chasing the Higgs shape at HL-LHC, Phys. Rev. D 104, 095021 (2021).
[73] K. Mimasu, V. Sanz, and C. Williams, Higher order QCD predictions for associated Higgs production with anomalous couplings to gauge bosons, J. High Energy Phys. 08 (2016) 039.

[74] F. Caola, K. Melnikov, and R. Röntsch, Nested softcollinear subtractions in NNLO QCD computations, Eur. Phys. J. C 77, 248 (2017).

[75] F. Caola, K. Melnikov, and R. Röntsch, Analytic results for color-singlet production at NNLO QCD with the nested soft-collinear subtraction scheme, Eur. Phys. J. C 79, 386 (2019).

[76] D. de Florian et al. (LHC Higgs Cross Section Working Group), Handbook of LHC Higgs cross sections: 4. Deciphering the nature of the Higgs sector, arXiv: 1610.07922 .

[77] K. G. Chetyrkin, J. H. Kühn, and M. Steinhauser, RunDec: A mathematica package for running and decoupling of the strong coupling and quark masses, Comput. Phys. Commun. 133, 43 (2000).

[78] F. Herren and M. Steinhauser, Version 3 of RunDec and CRunDec, Comput. Phys. Commun. 224, 333 (2018).

[79] M. Cacciari, G. P. Salam, and G. Soyez, The anti- $k_{t}$ jet clustering algorithm, J. High Energy Phys. 04 (2008) 063.

[80] M. Cacciari, G. P. Salam, and G. Soyez, FastJet user manual, Eur. Phys. J. C 72, 1896 (2012).

[81] C. Englert, M. McCullough, and M. Spannowsky, Gluoninitiated associated production boosts Higgs physics, Phys. Rev. D 89, 013013 (2014).

[82] M. Farina, C. Grojean, F. Maltoni, E. Salvioni, and A. Thamm, Lifting degeneracies in Higgs couplings using single top production in association with a Higgs boson, J. High Energy Phys. 05 (2013) 022.

[83] A. M. Sirunyan et al. (CMS Collaboration), Search for associated production of a Higgs boson and a single top quark in proton-proton collisions at $\sqrt{s}=13 \mathrm{TeV}$, Phys. Rev. D 99, 092005 (2019).

[84] K.-P. Xie and B. Yan, Probing the electroweak symmetry breaking with Higgs production at the LHC, Phys. Lett. B 820, 136515 (2021).

[85] A. Helset, A. Martin, and M. Trott, The geometric standard model effective field theory, J. High Energy Phys. 03 (2020) 163.

[86] G. Aad et al. (ATLAS Collaboration), Measurements of $W H$ and $Z H$ production in the $H \rightarrow b \bar{b}$ decay channel in $p p$ collisions at $13 \mathrm{TeV}$ with the ATLAS detector, Eur. Phys. J. C 81, 178 (2021).

[87] M. Cepeda et al., Report from working group 2: Higgs physics at the HL-LHC and HE-LHC, CERN Yellow Rep. Monogr. 7, 221 (2019). 